\title{
Effective asymmetric Michael addition of acetone to nitroalkenes promoted by chiral proline amide-thiourea bifunctional catalysts
}

\author{
Qiao-wei Wang, ${ }^{\text {a,b }}$ Lin Peng, ${ }^{\text {a,b }}$ Ji-ya Fu, ${ }^{\text {a,b }}$ Qing-chun Huang, Li-xin Wang, ${ }^{\text {a,* }}$ and Xiao- \\ ying $X \mathbf{u}^{\mathrm{a}} *$ \\ ${ }^{a}$ Key Laboratory of Asymmetric Synthesis \& Chirotechnology of Sichuan Province, Chengdu \\ Institute of Organic Chemistry, Chinese Academy of Sciences, Chengdu 610041, P.R. China \\ ${ }^{b}$ Graduate School of Chinese Academy of Sciences, Beijing 100039, P.R. China \\ Email:wlxioc@cioc.ac.cn xuxy@cioc.ac.cn
}

\begin{abstract}
A series of secondary amine-thiourea catalysts 1a-1d derived from L-proline and chiral diamine were prepared and successfully applied to the Michael addition of acetone to trans-nitroalkenes in excellent yields (up to 99\%) and enantioselectivities (44-91\% ee).
\end{abstract}

Keywords: Organocatalyst, Michael addition, acetone, trans-nitroalkenes, chiral proline amidethiourea

\section{Introduction}

Michael addition is one of the most important reactions in carbon-carbon bond formation. Particularly, Michael reaction involved trans-nitroalkenes and ketones is a convenient approach to $\gamma$-nitroketones which are valuable building blocks in organic synthesis and could be readily converted to commercially important pharmaceutical products via $\gamma$-aminobutyric acids. ${ }^{1-2}$ Over the past years, various efficient chiral organocatalysts have been developed for the enantioselective Michael addition of aldehydes, ${ }^{3}$ ketones $^{4}$ and 1,3-dicarbonyl compounds ${ }^{5}$ to nitroalkenes. In 2005, Jacobsen ${ }^{6}$ first reported the highly efficient primary amine-thiourea catalyzed addition of ketone to nitroalkenes in high enantioselectivities across a broad range of substrates, as well as high diastereo- and regioselectivities. In 2008, Zhao ${ }^{7}$ reported that the assemblies of simple $\alpha$-amino acids and quinine derived tertiary amine-thioureas can serve as excellent asymmetric catalysts for such an addition. Tsogoeva ${ }^{8}$ has developed imidazole based thioureas which exhibit good enantioselectivities in the same addition of acetone to several nitroalkenes. Although excellent results have been achieved by these systems, few excellent protocols of asymmetric Michael addition of acetone to nitroalkenes were reported. ${ }^{9}$ The successful design of a simple and highly effective chiral catalyst for the Michael addition of acetone to nitroolefins with excellent enantioselectivities is still a challenging task. 
Recently, bifunctional activations, which simultaneously activate both acceptors and donors, have been regarded as an important strategy in asymmetric small molecular catalysis. ${ }^{10}$ As a typical and effective activation model, chiral thiourea catalysts have been widely used due to their effective activation of carbonyl and nitro groups through double hydrogen-bonding interactions, ${ }^{11}$ and second amine, especially L-proline and L-prolic amides, have been well identified as powerful catalysts to activate aldehydes or ketones via enamine or imine transition state. ${ }^{12}$ Held the concept of bifunctional activations, we considered a kind of catalysts bearing both L-proline and thiourea functional moieties linked by a suitable chiral linker and expected they may simultaneously activate both a nucleophile and an electrophile in the same asymmetric reaction. Typically, the chiral proline amide-thiourea catalysts 1a-1d (Figure 1) in our hands, synergistically combining two catalytic sites of chiral thiourea and L-prolic amide skeleton didn't draw enough attention. ${ }^{13}$ We excepted that these bifunctional catalysts may catalyze the asymmetric Michael addition of acetone to nitroalkenes and the reactivity and enantioselectivity may be enhanced by double activation, mutual stereo-compatibility and chiral recognition.

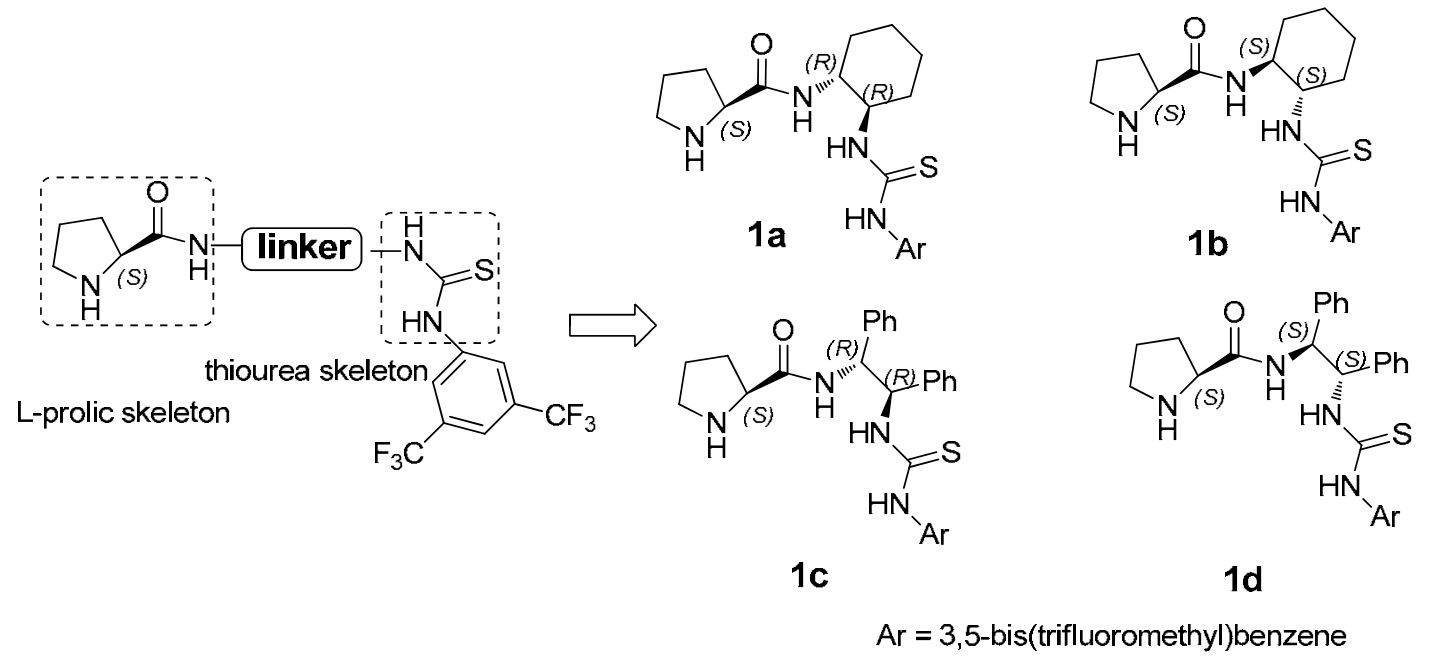

Figure 1. Chiral proline amide-thiourea bifunctional catalysts.

As a part of our continuing interests in asymmetric synthesis, ${ }^{14-15}$ herein, we wish to report the first example of these chiral proline amide-thiourea bifunctional catalysts promoted enantioselective Michael addition of acetone to nitroalkenes in good yields and enantioselectivities. The whole scheme, strategies for these catalysts are illustrated in Figure 1.

\section{Results and Discussion}

Chiral catalysts 1a-1d may be similarly prepared as reported procedures. ${ }^{13}$ To determine the optimal asymmetric reaction conditions, Michael addition of acetone to trans-nitrostyrene at 
room temperature was selected as the model reaction and chiral catalysts 1a-1d were initially screened, and the results were shown in Table 1. All catalysts of 1a-1d afforded good yields (65$89 \%$ ), whereas in disappointing enantioselectivities (5-36\% ee). Comparatively, catalysts 1a and 1c gave better yields and enantioselectivities (Table 1, entries 1 vs 2, 3 vs 4 ). It is probably due to the compatibility of the two catalytic chiral centers. The less incompatible chiral centers in $\mathbf{1 b}$ and 1d probably exert no synergic or negative effects on the enantioselectivity. Catalysts 1a, bearing a $(\mathrm{R}, \mathrm{R})$-linker, affording the desired products in $36 \%$ ee, was chosen for further optimization.

Table 1. The catalyst screening for Michael addition of acetone to trans-nitrostyrene ${ }^{\text {a }}$

\begin{tabular}{|c|c|c|c|c|}
\hline Entry & Cat. & Config $^{\mathrm{b}}$ & Yield $(\%)^{\mathrm{c}}$ & $\operatorname{Ee}(\%)^{\mathrm{d}}$ \\
\hline 1 & $1 \mathbf{a}$ & $\mathrm{S}$ & 89 & 36 \\
\hline 2 & $1 b$ & $\mathrm{~S}$ & 75 & 5 \\
\hline 3 & 1c & $\mathrm{S}$ & 78 & 26 \\
\hline 4 & 1d & $\mathrm{S}$ & 65 & 12 \\
\hline
\end{tabular}

${ }^{\mathrm{a}}$ The reaction was conducted with trans-nitrostyrene $(0.1 \mathrm{mmol})$ in $1 \mathrm{~mL}$ acetone at room temperature for 48 hours.

${ }^{\mathrm{b}}$ By comparison with the same compound reported.

${ }^{\mathrm{c}}$ Isolated yield after silica gel chromatography.

${ }^{\mathrm{d}}$ Determined by HPLC using Chiral Whelk-01 column.

With the selected catalyst 1a, effects of solvents and additives were investigated to optimize the reaction conditions. As shown in Table 2, the yields and enantioselectivities were highly variable in different solvents. In polar protic solvents such as $\mathrm{CH}_{3} \mathrm{OH}$, almost no desired product was observed after 72 hours (Table 2, entry 1), whereas in polar aprotic solvents such as DMF, the Michael reaction proceeded smoothly and gave the desired product in $88 \%$ yield and $<5 \%$ ee (Table 2, entry 2). Less polar solvents were better for this transformation and the Michael adducts were obtained in excellent yield (83-96\%) and moderate to good enantioselectivities (23$81 \%$ ee) (Table 2, entries 3-11). When $\mathrm{Et}_{2} \mathrm{O}$ or $\mathrm{THF}$ as solvent, the reaction was performed smoothly and relatively high yields and moderate to good enantioselectivities were obtained (Table 2 , entries 8,10$)$. In particular, the highest enantioselectivity $(81 \%$ ee) was achieved when $\mathrm{Et}_{2} \mathrm{O}$ used as solvent (Table 2, entry 10). To further increase the enantioselectivity, a series of additives such as $\mathrm{H}_{2} \mathrm{O}$, acids and bases were evaluated. $15 \mathrm{~mol} \%$ water gave racemic product (Table 2, entry 12) and 2 eq water afforded only $14 \%$ ee (Table 2, entry 13). The addition of acids (Table 2, entries 15, 16, 18-21) and bases (Table 2, entries 22-25) could not gave improved results. The influence of temperature was also investigated. Lower temperature can slightly increase the enantioselectivity $(91 \%$ ee), while dramatically decrease the reaction rate ( $21 \%$ yield, 
Table 2, entry 17). Through extensive screening, the optimized reaction conditions were found to be $20 \mathrm{~mol} \%$ of catalyst $\mathbf{1 a}, 20$ eq. acetone, $\mathrm{Et}_{2} \mathrm{O}$ as solvent at room temperature.

Table 2. Effects of solvents and additives ${ }^{a}$

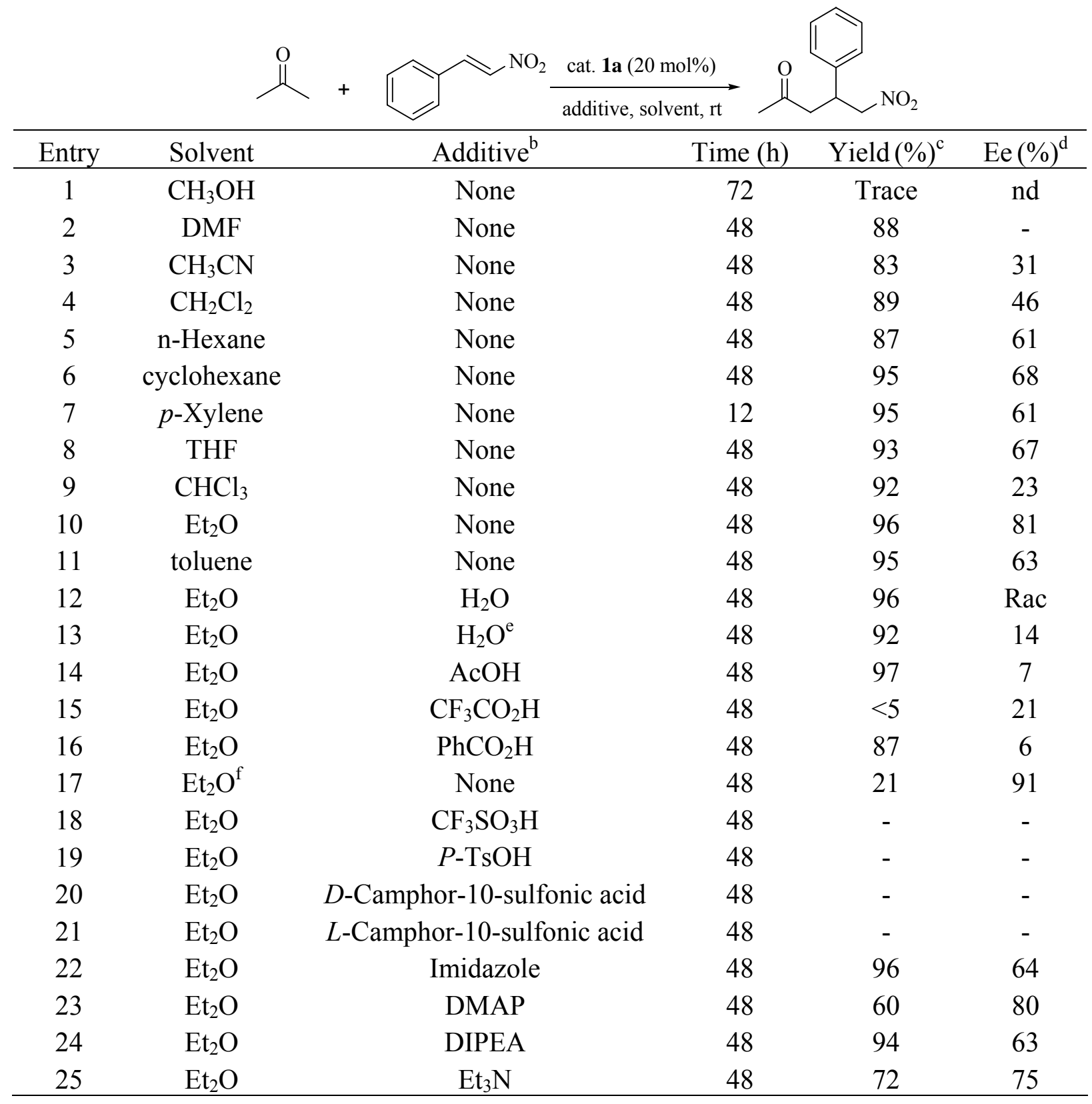

${ }^{a}$ Unless noted otherwise, the reaction was conducted with trans-nitrostyrene $(0.1 \mathrm{mmol}, 1 \mathrm{eq}$.$) ,$ acetone $(0.15 \mathrm{~mL}, 20$ eq. $)$, solvent $(0.85 \mathrm{~mL})$ and catalyst $1 \mathbf{a}(20 \mathrm{~mol} \%)$ and stirred at room temperature.

${ }^{\mathrm{b}} 15 \mathrm{~mol} \%$ additive loading unless stated otherwise.

${ }^{\mathrm{c}}$ Isolated yield after silica gel chromatography.

${ }^{\mathrm{d}}$ Determined by HPLC using Chiral Whelk-01 column. 
e 200 mol \% additive loading.

${ }^{\mathrm{f}}$ The reaction was performed at $-20{ }^{\circ} \mathrm{C}$.

Having established optimized reaction conditions for the model reaction, the scope of this transformation was studied and the results were listed in Table 3. Almost all the substituted $\beta$ nitroalkenes bearing either electron-donating or withdrawing substituents on the aromatic ring gave the desired Michael adducts in excellent yields (up to 99\%) and moderate to good enantioselectivities (44-84\% ee). Unsubstituted $\beta$-nitroalkenes gave better enantioselectivity compared with substituted $\beta$-nitroalkenes bearing electron-withdrawing or electron-donating groups in the phenyl ring (Table 3, entries 1 vs 2-6). When 2-(1-naphthyl)-nitroethene 3g used, the highest yield (99\%) and good enantioselectivity ( $76 \%$ ee) were achieved (Table 3, entry 7 ), however only moderate yield and enantioselectivity were obtained when the substituted napthylnitroethylene used (Table 3, entry 8). Further extending the optimized protocol to heteroaromatic nitroalkenes such as 2-furanyl-nitroethene and 2-thienyl-nitroethene, moderate to good yields and enantioselectivities (Table 3, entries 9-10) were observed. Relatively, 2-thienyl-nitroethene provided the highest enantioselectivity and excellent yield (84\% ee, $91 \%$ yield; Table 3, entry $10)$.

Table 3. Asymmetric Michael addition of acetone to $\beta$-nitroalkenes ${ }^{\text {a }}$<smiles>[CH2+]C(C)=O</smiles>

2

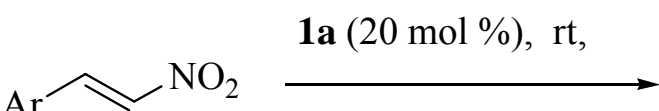

3
$\mathrm{Et}_{2} \mathrm{O}, 48 \mathrm{~h}$

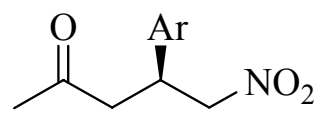

4

\begin{tabular}{cccc}
\hline Entry & $\mathrm{Ar}$ & Yield $(\%)^{\mathrm{b}}$ & Ee $(\%)^{\mathrm{c}}$ \\
\hline 1 & $\mathrm{Ph} \mathbf{3 a}$ & $96 \mathbf{4 a}$ & 81 \\
2 & $4-\mathrm{ClC}_{6} \mathrm{H}_{4} \mathbf{3 b}$ & $97 \mathbf{4 b}$ & 55 \\
3 & $4-\mathrm{CH}_{3} \mathrm{C}_{6} \mathrm{H}_{4} \mathbf{3 c}$ & $95 \mathbf{4 c}$ & 72 \\
4 & $3-\mathrm{FC}_{6} \mathrm{H}_{4} \mathbf{3 d}$ & $98 \mathbf{4 d}$ & 50 \\
5 & $3,4-\left(\mathrm{CH}_{3} \mathrm{O}\right)_{2} \mathrm{C}_{6} \mathrm{H}_{3} \mathbf{3 e}$ & $87 \mathbf{4 e}$ & 61 \\
6 & $3,4,5-\left(\mathrm{CH}_{3} \mathrm{O}\right)_{3} \mathrm{C}_{6} \mathrm{H}_{2} \mathbf{3 f}$ & $98 \mathbf{4 f}$ & 44 \\
7 & $1-\mathrm{Naphthyl} \mathbf{g}_{3}$ & $99 \mathbf{4 g}$ & 76 \\
8 & 6-CH & $62 \mathbf{4 h}$ & 59 \\
9 & 2-Furyl 3i & $95 \mathbf{4 i}$ & 56 \\
10 & 2-Thienyl 3j & $91 \mathbf{4 j}$ & 84 \\
\hline
\end{tabular}

${ }^{\mathrm{a}}$ Unless noted otherwise, the reaction was conducted with trans-nitroalkenes ( $0.1 \mathrm{mmol}$, 1eq.), acetone $(0.15 \mathrm{~mL}, 20$ eq. $)$, solvent $(0.85 \mathrm{~mL})$ and catalyst $1 \mathbf{a}(20 \mathrm{~mol} \%)$ and stirred at room temperature.

${ }^{\mathrm{b}}$ Isolated yield after silica gel chromatography.

${ }^{\mathrm{C}}$ Determined by HPLC using Chiral Whelk-01 column or chiralpak OD-H column.

\section{Conclusions}


In conclusion, we successfully applied the chiral proline amide-thiourea bifunctional catalysts 1a-1d with two catalytic sites of chiral thiourea and L-prolic amide skeleton to catalyze the Michael addition of acetone to nitroalkenes with excellent yields (up to $99 \%$ ) and enantioselectivities (up to $91 \%$ ee) for a variety of aryl and hetereoaryl nitroalkenes. Further applications of the newly developed catalysts and related analogues in other catalytic reactions are currently underway.

\section{Experimental Section}

General Procedures. ${ }^{1} \mathrm{H}$ NMR and ${ }^{13} \mathrm{C}$ NMR spectra were recorded on a Bruker NMR (300 MHz). Chemical shifts of ${ }^{1} \mathrm{H}$ and ${ }^{13} \mathrm{C}$ were given in $\delta$ relative to tetramethylsilane (TMS). Coupling constant $J$ was given in Hz. Enantioselectivities were determined by HPLC analysis on chiral Whelk-01 or Chiralpak OD-H columns. IR spectra were recorded on a ThermoFisher Nicolet 6700 FTIR spectrometer on a $\mathrm{KBr}$ beamsplitter. High-resolution mass spectra were obtained with the microTOF-Q II10203 mass spectrometer.

\section{Typical procedure for the preparation of catalyst 1}

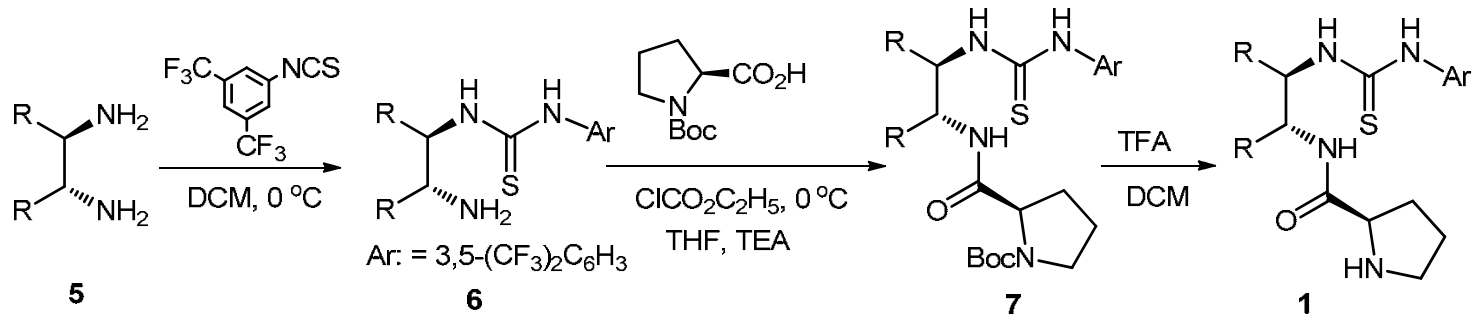

To a solution of chiral amine 5 (10.7 mmol, 1.0 eq) in $\mathrm{CH}_{2} \mathrm{Cl}_{2}(30 \mathrm{~mL})$, was added 3,5bis(trifuoromethyl)phenyl isothiocyanate $(2.33 \mathrm{~g}, 8.6 \mathrm{mmol}, 0.8 \mathrm{eq})$. The reaction mixture was stirred at $0{ }^{\circ} \mathrm{C}$ for 20 hours. After the reaction was completed (monitored by TLC), the solvent was removed under reduced pressure and the residue was purified by column chromatography on silica gel (eluent PE:EtOAc = 8:1 to EtOAc) to afford pure products 6 as a light yellow solid.

The solution of $(S)$-Boc-proline $(3.00 \mathrm{~g}, 14.1 \mathrm{mmol}, 1.1 \mathrm{eq})$, TEA $(1.41 \mathrm{~g}, 14.3 \mathrm{mmol}, 1.1 \mathrm{eq})$ in THF $(40 \mathrm{~mL})$ was stirred for $1 \mathrm{~h}$ at $0{ }^{\circ} \mathrm{C}$, and ethyl chloroformate $(1.25 \mathrm{~mL}, 14.3 \mathrm{mmol}, 1.1 \mathrm{eq})$ was added and stirred at $0{ }^{\circ} \mathrm{C}$ for $30 \mathrm{~min}$. Compound 6 (13.0 mmol, $\left.1.0 \mathrm{eq}\right)$ was then added, and the solution was stirred for another $12 \mathrm{~h}$ at $0{ }^{\circ} \mathrm{C}$. After the reaction was completed (monitored by TLC), the mixture was filtered, and the organic layer was removed under reduced pressure and the residue was purified by column chromatography on silica gel (eluent PE:EtOAc $=8: 1$ to EtOAc) to afford pure products 7. 
Trifluoroacetic acid $(9.0 \mathrm{~mL})$ was added dropwise to a solution of 7 (10.4 mmol) in $\mathrm{CH}_{2} \mathrm{Cl}_{2}$ $(20 \mathrm{~mL})$ at ambient temperature. The solution was stirred for 2 hours (monitored by TLC), and the $\mathrm{pH}$ value was adjusted to 8.0 by aqueous $\mathrm{NaHCO}_{3}$. The mixture was extracted with $\mathrm{CH}_{2} \mathrm{Cl}_{2}$ (30 mLx3), and the organic layer was combined and dried over anhydrous $\mathrm{MgSO}_{4}$. After filtration, the solution was concentrated in vacuum. The crude product was purified by column chromatography on silica gel (eluent PE:tOAc $=10: 1$ to EtOAc) to afford target catalyst $\mathbf{1}$.

(S)-N((1R,2R)-2-(3-(3,5-Bis(trifluoromethyl)phenyl)thioureido)cyclohexyl)pyrrolidine-2-

carboxamide 1 1a. $^{13}$ white solid in $82 \%$ yield; ${ }^{1} \mathrm{H}$ NMR $\left(300 \mathrm{MHz}^{\mathrm{CDCl}} 3\right): \delta 9.89(\mathrm{~s}, 1 \mathrm{H}, \mathrm{NH})$, $8.21(\mathrm{~d}, 1 \mathrm{H}, J=9.57 \mathrm{~Hz}, \mathrm{NH}), 8.12$ (s, 2H, Ar-H), 7.80 (s, 1H, NH), 7.55 (s, 1H, Ar-H), 4.63-4.60 $(\mathrm{m}, 1 \mathrm{H}, \mathrm{CHC}=\mathrm{O}), 3.74-3.67\left(\mathrm{~m}, 2 \mathrm{H}, \mathrm{NH}-\mathrm{CH}_{2}\right), 3.01-2.96(\mathrm{~m}, 2 \mathrm{H}, \mathrm{CHCH}), 1.99-1.94(\mathrm{~m}, 1 \mathrm{H}$, $\left.\mathrm{CH}_{2}\right), 1.63-1.70\left(\mathrm{~m}, 6 \mathrm{H}, \mathrm{CH}_{2}, 2 \mathrm{CH}_{2}, \mathrm{NH}\right), 1.47-1.55\left(\mathrm{~m}, 4 \mathrm{H},-\mathrm{CH}_{2} \mathrm{CH}_{2}-\right), 1.30-1.35\left(\mathrm{~m}, 2 \mathrm{H}, \mathrm{CH}_{2}\right)$ ppm; ${ }^{13} \mathrm{C}$ NMR $\left(75 \mathrm{MHz}, \mathrm{CDCl}_{3}\right): \delta 180.9(\mathrm{C}=\mathrm{S}), 176.7(\mathrm{C}=\mathrm{O}), 141.3$ (Cquat), 131.4 (Cquat), 126.8 (Cquat, $\left.\mathrm{CF}_{3}\right), 122.1(\mathrm{CH}), 117.0(\mathrm{CH}), 60.3(\mathrm{CH}), 56.8(\mathrm{CH}), 53.7(\mathrm{CH}), 47.1\left(\mathrm{CH}_{2}\right), 32.9$ $\left(\mathrm{CH}_{2}\right), 32.2\left(\mathrm{CH}_{2}\right), 30.0\left(\mathrm{CH}_{2}\right), 25.9\left(\mathrm{CH}_{2}\right), 25.4\left(\mathrm{CH}_{2}\right), 24.7\left(\mathrm{CH}_{2}\right)$ ppm; HRMS (ESI-TOF) calcd for $\mathrm{C}_{20} \mathrm{H}_{25} \mathrm{~F}_{6} \mathrm{~N}_{4} \mathrm{OS}\left([\mathrm{M}+\mathrm{H}]^{+}\right)$: 483.1648 , found: 483.1660 .

(S)-N((1S,2S)-2-(3-(3,5-Bis(trifluoromethyl)phenyl)thioureido)cyclohexyl)pyrrolidine-2-

carboxamide 1 b. $^{13}$ white solid, 64\% overall yield. 9.90 (br, $\left.1 \mathrm{H}, \mathrm{NH}\right), 8.21$ (d, $J=9.84 \mathrm{~Hz}, 1 \mathrm{H}$, $\mathrm{NH}), 8.13$ (s, 2H, Ar-H) 7.79 (d, J=8.98 Hz, 1H, NH), 7.55 (s, 1H, Ar-H ), 4.59-4.62 (m, 1H, $\mathrm{CHC}=\mathrm{O}), 3.69-3.73\left(\mathrm{~m}, 2 \mathrm{H}, \mathrm{NH}-\mathrm{CH}_{2}\right), 2.97-3.03(\mathrm{~m}, 2 \mathrm{H}, \mathrm{CHCH}), 2.10-2.40\left(\mathrm{~m}, 1 \mathrm{H}, \mathrm{CH}_{2}\right)$, 1.89-2.01 (m, 6H, $\left.\mathrm{CH}_{2}, 2 \mathrm{CH}_{2}, \mathrm{NH}\right), 1.30-1.70\left(\mathrm{~m}, 6 \mathrm{H}, 3 \mathrm{CH}_{2}\right),{ }^{13} \mathrm{C} \mathrm{NMR}\left(75 \mathrm{MHz}, \mathrm{CDCl}_{3}\right), \delta$ $181.0(\mathrm{C}=\mathrm{S}), 176.7(\mathrm{C}=\mathrm{O}), 141.4$ (Cquat), 131.5 (Cquat), 123.2 (Cquat, $\left.\mathrm{CF}_{3}\right), 122.1(\mathrm{CH}), 117.1$ $(\mathrm{CH}), 60.3(\mathrm{CH}), 56.9(\mathrm{CH}), 53.7(\mathrm{CH}), 47.1\left(\mathrm{CH}_{2}\right), 32.9\left(\mathrm{CH}_{2}\right), 32.3\left(\mathrm{CH}_{2}\right), 31.0\left(\mathrm{CH}_{2}\right), 25.9$ $\left(\mathrm{CH}_{2}\right), 25.4\left(\mathrm{CH}_{2}\right), 24.7\left(\mathrm{CH}_{2}\right)$ HRMS (ESI-TOF) calcd for $\mathrm{C}_{20} \mathrm{H}_{25} \mathrm{~F}_{6} \mathrm{~N}_{4} \mathrm{OS}\left([\mathrm{M}+\mathrm{H}]^{+}\right): 483.1648$, found: 483.1660 .

(S)-N-((1R,2R)-2-(3-(3,5-Bis(trifluoromethyl)phenyl)thioureido)-1,2-diphenylethyl)pyrrolidine-2-carboxamide 1c. White solid; $72.5 \%$ overall yield; ${ }^{1} \mathrm{H} \mathrm{NMR}\left(300 \mathrm{MHz} \mathrm{CDCl}_{3}\right): \delta 10.11$ (m, 1H, NH), 8.97 (d, J=9.96 Hz,1H, NH), 8.53 (d, J=8.94 Hz,1H, NH), 8.09 (s, 2H, ArH), 7.59 (s, 1H, ArH), 7.11-7.33 (m, $10 \mathrm{H}, \mathrm{ArH}), 6.44-6.47$ (m, 1H, CHCH), 5.41-5.48 (m, 1H, CHCH), 3.67-3.71 (m, 1H, CHNH), 3.12-3.15 (m, 1H, $\left.\mathrm{NHCH}_{2}\right), 2.98-3.03\left(\mathrm{~m}, 1 \mathrm{H}, \mathrm{CH}_{2}\right), 1.60-1.96(\mathrm{~m}$, $\left.4 \mathrm{H}, 2 \mathrm{CH}_{2}\right) ; \delta 181.5(\mathrm{C}=\mathrm{S}), 176.5(\mathrm{C}=\mathrm{O}), 141.0$ (Cquat), 138.0 (Cquat), 131.7 (Cquat), 123.2 (Cquat, $\left.\mathrm{CF}_{3}\right), 129.0(\mathrm{CH}), 128.6(\mathrm{CH}), 128.4(\mathrm{CH}), 127.9(\mathrm{CH}), 127.7(\mathrm{CH}), 122.9(\mathrm{CH}), 122.1$ $(\mathrm{CH}), 117.7(\mathrm{CH}), 61.9(\mathrm{CH}), 60.5(\mathrm{CH}), 58.6(\mathrm{CH}), 47.2\left(\mathrm{CH}_{2}\right), 31.1\left(\mathrm{CH}_{2}\right), 26.1\left(\mathrm{CH}_{2}\right)$.ppm; HRMS (ESI-TOF) calcd for $\mathrm{C}_{28} \mathrm{H}_{27} \mathrm{~F}_{6} \mathrm{~N}_{4} \mathrm{OS}\left([\mathrm{M}+\mathrm{H}]^{+}\right): 581.1804$, found: 581.1812 .

(S)-N-((1S,2S)-2-(3-(3,5-Bis(trifluoromethyl)phenyl)thioureido)-1,2-diphenylethl)pyrrolidine- 2 -carboxamide 1d. ${ }^{1} \mathrm{H}$ NMR (300 MHz, DMSO): $\delta$ (ppm): 9.78 (brs, $\left.1 \mathrm{H}, \mathrm{NH}\right), 8.75$ (brs, 1H, NH), 8.72 (brs, 1H, NH), 8.02 (s, 2H, Ar-H), 7.61(s, 1H, Ar-H), 7.18-7.28 (m, 10H, Ar-H), 6.23-6.25 (m, 1H, CHCH), 5.35-5.41 (m, 1H, CHCH), 3.66-3.69 (m, 1H, NHCH), 2.82-2.95 (m, $\left.2 \mathrm{H}, \mathrm{CH}_{2}\right), 2.14$ (brs, $\left.1 \mathrm{H}, \mathrm{NH}\right), 1.91-1.94\left(\mathrm{~m}, 1 \mathrm{H}, \mathrm{CH}_{2}\right), 1.53-1.72\left(\mathrm{~m}, 3 \mathrm{H}, \mathrm{CH}_{2}\right) . \delta 181.6(\mathrm{C}=\mathrm{S})$, $176.4(\mathrm{C}=\mathrm{O}), 140.8$ (Cquat), 137.8 (Cquat), 131.8 (Cquat), $129.0(\mathrm{CH}), 128.6(\mathrm{CH}), 128.2(\mathrm{CH})$, $127.9(\mathrm{CH}), 127.7(\mathrm{CH}), 127.5(\mathrm{CH}), 123.1\left(\mathrm{Cquat}, \mathrm{CF}_{3}\right), 123.4(\mathrm{CH}), 122.1(\mathrm{CH}), 118.0(\mathrm{CH})$, 
$62.9(\mathrm{CH}), 60.6(\mathrm{CH}), 59.4(\mathrm{CH}), 46.9\left(\mathrm{CH}_{2}\right), 30.4\left(\mathrm{CH}_{2}\right), 25.7\left(\mathrm{CH}_{2}\right)$.ppm; HRMS (ESI-TOF) calcd for $\mathrm{C}_{28} \mathrm{H}_{27} \mathrm{~F}_{6} \mathrm{~N}_{4} \mathrm{OS}\left([\mathrm{M}+\mathrm{H}]^{+}\right)$: 581.1804 , found: 581.1823 .

\section{Typical procedure for the asymmetric Michael addition of acetone to nitroalkenes}

Catalyst 1a $(0.02 \mathrm{mmol})$ was added to a stirred solution of acetone $2(2 \mathrm{mmol})$ in solvent $(0.85 \mathrm{~mL})$ under an atmosphere of air. The resulting solution was stirred for $5 \mathrm{~min}$ prior to the addition of nitroolefin $3(0.1 \mathrm{mmol})$ and the additive $(0.02 \mathrm{mmol})$. After stirring for the indicated reaction time at room temperature (monitored by TLC), the crude adduct was purified by column chromatography (petroleum ether/ethyl acetate, 10:1).

(4S)-5-Nitro-4-phenyl-pentan-2-one 4 ar. $^{9 \mathrm{f}}$ White solid; Yield 96\%; 81\% ee, chiral Whelk-01 column, $\mathrm{n}$-hexane $/ i$-PrOH $=80 / 20,1.0 \mathrm{ml} / \mathrm{min}, \lambda=220 \mathrm{~nm}, \mathrm{t}_{\mathrm{R}(\text { major })}=20.5 \mathrm{~min}, \mathrm{t}_{\mathrm{R}(\text { minor })}=16.7$ min, ${ }^{1} \mathrm{H}$ NMR $\left(300 \mathrm{MHz}, \mathrm{CDCl}_{3}\right): \delta 7.36-7.20(\mathrm{~m}, 5 \mathrm{H}, \mathrm{Ar}-\mathrm{H}), 4.70(\mathrm{dd}, J=6.9,12.3 \mathrm{~Hz}, 1 \mathrm{H}$, $\left.\mathrm{CH}_{2} \mathrm{NO}_{2}\right), 4.60\left(\mathrm{dd}, J=7.6,12.3 \mathrm{~Hz}, 1 \mathrm{H}, \mathrm{CH}_{2} \mathrm{NO}_{2}\right), 4.05-3.98(\mathrm{~m}, 1 \mathrm{H}, \mathrm{ArCH}), 2.92(\mathrm{~d}, J=7.0$ $\left.\mathrm{Hz}, 2 \mathrm{H}, \mathrm{CH}_{2}\right), 2.12\left(\mathrm{~s}, 3 \mathrm{H}, \mathrm{CH}_{3}\right) \mathrm{ppm} ;{ }^{13} \mathrm{C} \mathrm{NMR}\left(75 \mathrm{MHz}, \mathrm{CDCl}_{3}\right): \delta 205.3(\mathrm{C}=\mathrm{O}), 138.8$ (Cquat), 129.1(2CH), 127.9(CH), 127.4(2CH), 79.4 $\left(\mathrm{CH}_{2}\right), 46.1\left(\mathrm{CH}_{2}\right), 39.0(\mathrm{CH}), 30.4\left(\mathrm{CH}_{3}\right) \mathrm{ppm}$.

(4S)-5-Nitro-4-(4-chlorophenyl)-pentan-2-one $4 \mathrm{~b} .{ }^{9 \mathrm{f}}$ White solid; Yield 97\%; 55\% ee, chiral Whelk-01 column, $\mathrm{n}$-hexane $/ \mathrm{i}-\mathrm{PrOH}=80 / 20,1.0 \mathrm{ml} / \mathrm{min}, \lambda=220 \mathrm{~nm}, \mathrm{t}_{\mathrm{R} \text { (major) }}=19.2 \mathrm{~min}, \mathrm{t}_{\mathrm{R} \text { (minor) }}$ $=14.2 \mathrm{~min},{ }^{1} \mathrm{H}$ NMR $\left(300 \mathrm{MHz}, \mathrm{CDCl}_{3}\right): \delta$ 7.31-7.26 (m, 2H, Ar-H), 7.17-7.14 (m, 2H, Ar-H), 4.67 (dd, $J=6.6,12.5 \mathrm{~Hz}, 1 \mathrm{H}, \mathrm{CH}_{2} \mathrm{NO}_{2}$ ), 4.56 (dd, $J=8.0,12.5 \mathrm{~Hz}, 1 \mathrm{H}, \mathrm{CH}_{2} \mathrm{NO}_{2}$ ), 4.01-3.93 (m, $1 \mathrm{H}, \mathrm{ArCH}), 2.88\left(\mathrm{~d}, J=7.0 \mathrm{~Hz}, 2 \mathrm{H}, \mathrm{CH}_{2}\right), 2.12\left(\mathrm{~s}, 3 \mathrm{H}, \mathrm{CH}_{3}\right) \mathrm{ppm} ;{ }^{13} \mathrm{C} \mathrm{NMR}\left(75 \mathrm{MHz}, \mathrm{CDCl}_{3}\right): \delta$ 205.0(C=O), 137.7 (Cquat), 133.8 (Cquat), 129.2(CH), 128.8(CH), 79.1 $\left(\mathrm{CH}_{2}\right), 45.9\left(\mathrm{CH}_{2}\right)$, $38.3(\mathrm{CH}), 30.3 \mathrm{ppm}\left(\mathrm{CH}_{3}\right)$.

(4S)-5-Nitro-4-(4-methylphenyl)-pentan-2-one $\quad$ 4c. $^{9 \mathrm{f}}$ White solid; Yield 95\%; 72\% ee, chiralpak OD-H column, $\mathrm{n}$-hexane $/ i-\mathrm{PrOH}=80 / 20,0.7 \mathrm{ml} / \mathrm{min}, \lambda=220 \mathrm{~nm}, \mathrm{t}_{\mathrm{R} \text { (major) }}=19.2 \mathrm{~min}$, $\mathrm{t}_{\mathrm{R}(\text { minor })}=16.4 \mathrm{~min},{ }^{1} \mathrm{H}$ NMR $\left(300 \mathrm{MHz}, \mathrm{CDCl}_{3}\right): \delta$ 7.26-7.08 (m, 4H, Ar-H), 4.67 (dd, J=6.9, $12.2 \mathrm{~Hz}, 1 \mathrm{H}, \mathrm{CH}_{2} \mathrm{NO}_{2}$ ), 4.57 (dd, $J=7.6,12.2 \mathrm{~Hz}, 1 \mathrm{H}, \mathrm{CH}_{2} \mathrm{NO}_{2}$ ), 4.0-3.91 (m, $\left.1 \mathrm{H}, \mathrm{ArCH}\right), 2.89$ $\left(\mathrm{d}, J=7.0 \mathrm{~Hz}, 2 \mathrm{H}, \mathrm{CH}_{2}\right), 2.31\left(\mathrm{~s}, 3 \mathrm{H}, \mathrm{ArCH}_{3}\right), 2.11\left(\mathrm{~s}, 3 \mathrm{H}, \mathrm{CH}_{3}\right) \mathrm{ppm} ;{ }^{13} \mathrm{C} \mathrm{NMR}(75 \mathrm{MHz}$, $\left.\mathrm{CDCl}_{3}\right): \quad \delta \quad 206.5(\mathrm{C}=\mathrm{O}), \quad 138.5(\mathrm{Cquat}), \quad 136.7(2 \mathrm{CH}), \quad 130.7(\mathrm{CH}), \quad 128.2(2 \mathrm{CH}), \quad 80.6\left(\mathrm{CH}_{2}\right)$, 47.2( $\mathrm{CH}), 39.7\left(\mathrm{CH}_{2}\right), 31.3\left(\mathrm{CH}_{3}\right), 22.0\left(\mathrm{CH}_{3}\right) \mathrm{ppm}$.

(4S)-5-Nitro-4-(3-fluorophenyl)-pentan-2-one 4d. Yellow oil; Yield 98\%; 50\% ee, chiral Whelk-01 column, $\mathrm{n}$-hexane $/ i$-PrOH $=80 / 20,1.0 \mathrm{ml} / \mathrm{min}, \lambda=220 \mathrm{~nm}, \mathrm{t}_{\mathrm{R}(\text { major })}=14.6 \mathrm{~min}, \mathrm{t}_{\mathrm{R} \text { (minor) }}$ $=12.3 \mathrm{~min},{ }^{1} \mathrm{H}$ NMR $\left(300 \mathrm{MHz}, \mathrm{CDCl}_{3}\right): \delta$ 7.29-7.24(m, 1H, Ar-H), 7.01-6.98 (m, 1H, Ar-H), 6.94-6.91 (m, 2H, Ar-H), $4.68\left(\mathrm{dd}, J=6.5,12.5 \mathrm{~Hz}, 1 \mathrm{H}, \mathrm{CH}_{2} \mathrm{NO}_{2}\right), 4.57$ (dd, $J=8.0,12.5 \mathrm{~Hz}$, $\left.1 \mathrm{H}, \mathrm{CH}_{2} \mathrm{NO}_{2}\right), 4.02-3.95(\mathrm{~m}, 1 \mathrm{H}, \mathrm{ArCH}), 2.89\left(\mathrm{~d}, J=6.9 \mathrm{~Hz}, 2 \mathrm{H}, \mathrm{CH}_{2}\right), 2.10\left(\mathrm{~s}, 3 \mathrm{H}, \mathrm{CH}_{3}\right) \mathrm{ppm}$;

${ }^{13} \mathrm{C} \mathrm{NMR}\left(75 \mathrm{MHz}, \mathrm{CDCl}_{3}\right): \delta 205.0(\mathrm{C}=\mathrm{O}), 162.9$ (Cquat), 141.4 (Cquat), $130.6(\mathrm{CH})$, 123.9(CH), 114.8(CH), $114.4(\mathrm{CH}), 78.9\left(\mathrm{CH}_{2}\right), 45.6\left(\mathrm{CH}_{2}\right), 38.5(\mathrm{CH}), 30.2\left(\mathrm{CH}_{3}\right) \mathrm{ppm}$; IR (film, $\mathrm{cm}^{-1}$ ): $v$ 3066, 2964, 2920, 1717, 1615, 1592, 1556, 1490, 1452, 1379, 1248, 1165, 1146, 901, 789, 697, 523; HRMS (ESI-TOF) calcd for $\mathrm{C}_{11} \mathrm{H}_{12} \mathrm{FNNaO}_{3}\left([\mathrm{M}+\mathrm{Na}]^{+}\right)=248.0693$, Found 248.0699. 
(4S)-5-Nitro-4-(3, 4-dimethoxyphenyl))-pentan-2-one 4e. White solid; Yield 87\%; 61\% ee, chiralpak OD-H column, $\mathrm{n}$-hexane $/ i-\mathrm{PrOH}=80 / 20,0.7 \mathrm{ml} / \mathrm{min}, \lambda=220 \mathrm{~nm}, \mathrm{t}_{\mathrm{R} \text { (major) }}=40.2 \mathrm{~min}$, $\mathrm{t}_{\mathrm{R}(\text { minor })}=34.5 \mathrm{~min},{ }^{1} \mathrm{H}$ NMR $\left(300 \mathrm{MHz}, \mathrm{CDCl}_{3}\right): \delta 6.82-6.80(\mathrm{~m}, 1 \mathrm{H}, \mathrm{Ar}-\mathrm{H}), 6.76-6.72(\mathrm{~m}, 2 \mathrm{H}$, Ar-H), 4.67 (dd, $J=6.9,12.2 \mathrm{~Hz}, 1 \mathrm{H}, \mathrm{CH}_{2} \mathrm{NO}_{2}$ ), 4.57 (dd, $J=7.6,12.2 \mathrm{~Hz}, 1 \mathrm{H}, \mathrm{CH}_{2} \mathrm{NO}_{2}$ ), $4.00-$ $3.90(\mathrm{~m}, 1 \mathrm{H}, \mathrm{ArCH}), 3.87\left(\mathrm{~s}, 3 \mathrm{H}, \mathrm{OCH}_{3}\right), 3.85\left(\mathrm{~s}, 3 \mathrm{H}, \mathrm{OCH}_{3}\right), 2.89$ (d, J=7.0 Hz, $\left.2 \mathrm{H}, \mathrm{CH}_{2}\right), 2.12$ (s, 3H, $\mathrm{CH}_{3}$ ) ppm; ${ }^{13} \mathrm{C}$ NMR (75 MHz, $\mathrm{CDCl}_{3}$ ): $\delta 205.5$ (C=O), 149.2 (Cquat), 148.6 (Cquat), 131.2 (Cquat), $119.1(\mathrm{CH}), 111.5(\mathrm{CH}), 110.9(\mathrm{CH}), 79.6\left(\mathrm{CH}_{2}\right), 55.9\left(\mathrm{OCH}_{3}\right), 46.3\left(\mathrm{CH}_{2}\right), 38.8$ (CH), $30.4\left(\mathrm{CH}_{3}\right)$ ppm; IR (film, $\left.\mathrm{cm}^{-1}\right): v 3005,2964,2841,1717,1543,1443,1362,1262,1147$, 1022, 870, 816, 766, 642; HRMS (ESI-TOF) calcd for $\mathrm{C}_{13} \mathrm{H}_{17} \mathrm{NNaO}_{5}\left([\mathrm{M}+\mathrm{Na}]^{+}\right)=290.0999$, Found 290.1011.

(4S)-5-Nitro-4-(3,4,5-trimethoxyphenyl))-pentan-2-one 4f. White solid; Yield 98\%; 44\% ee, chiralpak OD-H column, $\mathrm{n}$-hexane $/ i-\mathrm{PrOH}=80 / 20,0.9 \mathrm{ml} / \mathrm{min}, \lambda=220 \mathrm{~nm}, \mathrm{t}_{\mathrm{R}(\text { major) }}=34.7 \mathrm{~min}$, $\mathrm{t}_{\mathrm{R}(\text { minor) }}=29.6 \mathrm{~min},{ }^{1} \mathrm{H}$ NMR $\left(300 \mathrm{MHz}, \mathrm{CDCl}_{3}\right): \delta 6.40(\mathrm{~s}, 2 \mathrm{H}, \mathrm{Ar}-\mathrm{H}), 4.68(\mathrm{dd}, J=6.9,12.3 \mathrm{~Hz}$, $\left.1 \mathrm{H}, \mathrm{CH}_{2} \mathrm{NO}_{2}\right), 4.59\left(\mathrm{dd}, J=7.6,12.3 \mathrm{~Hz}, 1 \mathrm{H}, \mathrm{CH}_{2} \mathrm{NO}_{2}\right), 4.97-3.88(\mathrm{~m}, 1 \mathrm{H}, \mathrm{ArCH}), 3.85(\mathrm{~s}, 6 \mathrm{H}$, $\left.\mathrm{OCH}_{3}\right), 3.81\left(\mathrm{~s}, 3 \mathrm{H}, \mathrm{OCH}_{3}\right), 2.90\left(\mathrm{~d}, J=6.9 \mathrm{~Hz}, 2 \mathrm{H}, \mathrm{CH}_{2}\right), 2.14\left(\mathrm{~s}, 3 \mathrm{H}, \mathrm{CH}_{3}\right) \mathrm{ppm} ;{ }^{13} \mathrm{C} \mathrm{NMR}(75$ $\left.\mathrm{MHz}, \mathrm{CDCl}_{3}\right): \delta 205.3(\mathrm{C}=\mathrm{O}), 153.5$ (Cquat), 134.5 (Cquat), $104.4(\mathrm{CH}), 79.3\left(\mathrm{CH}_{2}\right), 56.2$ $\left(\mathrm{OCH}_{3}\right), 46.3\left(\mathrm{CH}_{2}\right), 39.3(\mathrm{CH}), 30.4\left(\mathrm{CH}_{3}\right) \mathrm{ppm}$; IR (film, $\left.\mathrm{cm}^{-1}\right): v$ 3086, 3005, 2964, 2916, 2841, 1721, 1594, 1545, 1429, 1372, 1321, 1254, 1019, 1173, 1125, 998, 775, 675, 600; HRMS (ESITOF) calcd for $\mathrm{C}_{14} \mathrm{H}_{19} \mathrm{NNaO}_{6}\left([\mathrm{M}+\mathrm{Na}]^{+}\right)=320.1105$, Found 320.1106 .

(4S)-5-Nitro-4-(1-naphthalenyl))-pentan-2-one 4g. ${ }^{16}$ Yellow oil; Yield 99\%; 76\% ee, chiral Whelk-01 column, $\mathrm{n}$-hexane $/ i-\mathrm{PrOH}=80 / 20,1.0 \mathrm{ml} / \mathrm{min}, \lambda=220 \mathrm{~nm}, \mathrm{t}_{\mathrm{R}(\text { major })}=29.4 \mathrm{~min}, \mathrm{t}_{\mathrm{R}(\text { minor) }}$ $=17.7 \mathrm{~min},{ }^{1} \mathrm{H}$ NMR $\left(300 \mathrm{MHz}, \mathrm{CDCl}_{3}\right): \delta 8.18(\mathrm{~d}, J=8.5 \mathrm{~Hz}, 1 \mathrm{H}, \mathrm{Ar}-\mathrm{H}), 7.88(\mathrm{~d}, J=7.9 \mathrm{~Hz}$, $1 \mathrm{H}, \operatorname{Ar}-\mathrm{H}), 7.78(\mathrm{~d}, J=8.2 \mathrm{~Hz}, 1 \mathrm{H}, \mathrm{Ar}-\mathrm{H}), 7.62-7.50$ (m, 2H, Ar-H), 7.45-7.39 (m, 1H, Ar-H), $7.32(\mathrm{~d}, J=7.1 \mathrm{~Hz}, 1 \mathrm{H}, \mathrm{Ar}-\mathrm{H}), 4.97-4.90(\mathrm{~m}, 1 \mathrm{H}, \mathrm{ArCH}), 4.80-4.75\left(\mathrm{~m}, 2 \mathrm{H}, \mathrm{CH}_{2} \mathrm{NO}_{2}\right), 3.08-$ $3.04\left(\mathrm{~m}, 2 \mathrm{H}, \mathrm{CH}_{2}\right), 2.10\left(\mathrm{~s}, 3 \mathrm{H}, \mathrm{CH}_{3}\right) \mathrm{ppm} ;{ }^{13} \mathrm{C} \mathrm{NMR}\left(75 \mathrm{MHz}, \mathrm{CDCl}_{3}\right): \delta 205.5(\mathrm{C}=\mathrm{O}), 134.8$ (Cquat), 134.0 (Cquat), 130.8 (Cquat), $129.1(\mathrm{CH}), 128.3(\mathrm{CH}), 126.8(\mathrm{CH}), 126.0(\mathrm{CH}), 125.2$ $(\mathrm{CH}), 123.5(\mathrm{CH}), 122.2(\mathrm{CH}), 78.7\left(\mathrm{CH}_{2}\right), 45.9\left(\mathrm{CH}_{2}\right), 33.3(\mathrm{CH}), 30.1\left(\mathrm{CH}_{3}\right) \mathrm{ppm}$.

(4S)-5-Nitro-4-(5-methoxy-1-naphthalenyl)-pentan-2-one 4h. White solid; Yield 62\%; 59\% ee, chiralpak OD-H column, $\mathrm{n}$-hexane $/ i-\mathrm{PrOH}=80 / 20,0.9 \mathrm{ml} / \mathrm{min}, \lambda=220 \mathrm{~nm}, \mathrm{t}_{\mathrm{R} \text { (major) }}=31.2 \mathrm{~min}$, $\mathrm{t}_{\mathrm{R}(\text { minor })}=26.6 \mathrm{~min},{ }^{1} \mathrm{H} \mathrm{NMR}\left(300 \mathrm{MHz}, \mathrm{CDCl}_{3}\right): \delta 7.73-7.67(\mathrm{~m}, 2 \mathrm{H}, \mathrm{Ar}-\mathrm{H}), 7.59(\mathrm{~s}, 1 \mathrm{H}, \mathrm{Ar}-\mathrm{H})$, 7.31-7.26 (m, 2H, Ar-H), 7.17-7.10 (m, 1H, Ar-H), 4.79-4.64 (m, 2H, $\left.\mathrm{CH}_{2} \mathrm{NO}_{2}\right), 4.19-4.09(\mathrm{~m}$, $1 \mathrm{H}, \mathrm{Ar}-\mathrm{H}), 3.91\left(\mathrm{~s}, 3 \mathrm{H}, \mathrm{OCH}_{3}\right), 2.99\left(\mathrm{~d}, J=7.0 \mathrm{~Hz}, 2 \mathrm{H}, \mathrm{CH}_{2}\right), 2.12\left(\mathrm{~s}, 3 \mathrm{H}, \mathrm{CH}_{3}\right) \mathrm{ppm} ;{ }^{13} \mathrm{C} \mathrm{NMR}$ $\left(75 \mathrm{MHz}, \mathrm{CDCl}_{3}\right): \delta 205.4(\mathrm{C}=\mathrm{O}), 158.0$ (Cquat), 134.0 (Cquat), 133.8 (Cquat), $129.3(\mathrm{CH})$, 128.9 (Cquat), $127.8(\mathrm{CH}), 126.3(\mathrm{CH}), 125.5(\mathrm{CH}), 119.4(\mathrm{CH}), 105.7(\mathrm{CH}), 79.5\left(\mathrm{CH}_{2}\right)$, $55.3\left(\mathrm{OCH}_{3}\right), 46.3\left(\mathrm{CH}_{2}\right), 39.1(\mathrm{CH}), 30.4\left(\mathrm{CH}_{3}\right) \mathrm{ppm}$; IR (film, $\left.\mathrm{cm}^{-1}\right): v$ 3066, 3020, 2919, 2958, 2922, 2850, 1718, 1606, 1550, 1384, 1363, 1233, 1165, 1025, 860, 815, 671, 569; HRMS (ESITOF) calcd for $\mathrm{C}_{16} \mathrm{H}_{17} \mathrm{NNaO}_{4}\left([\mathrm{M}+\mathrm{Na}]^{+}\right)=310.1050$, Found 310.1047 .

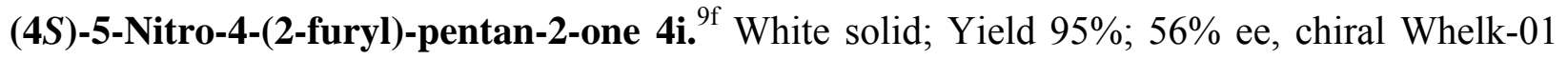
column, $\mathrm{n}$-hexane $/ i-\mathrm{PrOH}=80 / 20,1.0 \mathrm{ml} / \mathrm{min}, \lambda=220 \mathrm{~nm}, \mathrm{t}_{\mathrm{R}(\text { major })}=11.3 \mathrm{~min}, \mathrm{t}_{\mathrm{R}(\text { minor })}=10.5$ min, ${ }^{1} \mathrm{H}$ NMR (75 MHz, $\left.\mathrm{CDCl}_{3}\right): \delta$ 7.32-7.31 (m, 1H, Ar-H), 6.29-6.26 (m, 1H, Ar-H), 6.12-6.11 
$(\mathrm{m}, 1 \mathrm{H}), \mathrm{Ar}-\mathrm{H}, 4.66-4.64\left(\mathrm{~m}, 2 \mathrm{H}, \mathrm{CH}_{2} \mathrm{NO}_{2}\right), 4.10-4.05(\mathrm{~m}, 1 \mathrm{H}, \mathrm{Ar}-\mathrm{H}), 2.96$ (dd, $J=6.5,18.1$ $\left.\mathrm{Hz}, 1 \mathrm{H}, \mathrm{CH}_{2}\right), 2.87\left(\mathrm{dd}, J=7.3,18.1 \mathrm{~Hz}, 1 \mathrm{H}, \mathrm{CH}_{2}\right), 2.15\left(\mathrm{~s}, 3 \mathrm{H}, \mathrm{CH}_{3}\right) \mathrm{ppm} ;{ }^{13} \mathrm{C} \mathrm{NMR}(75 \mathrm{MHz}$, $\left.\mathrm{CDCl}_{3}\right): \delta 205.1(\mathrm{C}=\mathrm{O}), 151.7$ (Cquat), $142.3(\mathrm{CH}), 110.5(\mathrm{CH}), 107.0(\mathrm{CH}), 77.1\left(\mathrm{CH}_{2}\right), 43.4$ $\left(\mathrm{CH}_{2}\right), 32.8(\mathrm{CH}), 30.1\left(\mathrm{CH}_{3}\right) \mathrm{ppm}$.

(4S)-5-Nitro-4-(2-thiophenyl)-pentan-2-one 4j. ${ }^{6}$ Yellow oil; Yield 91\%; 84\% ee, chiral Whelk01 column, $\mathrm{n}$-hexane $/ i$-PrOH $=80 / 20,1.0 \mathrm{ml} / \mathrm{min}, \lambda=220 \mathrm{~nm}, \mathrm{t}_{\mathrm{R}(\text { major })}=14.2 \mathrm{~min}, \mathrm{t}_{\mathrm{R}(\text { minor })}=12.7$ min, ${ }^{1} \mathrm{H}$ NMR $\left(300 \mathrm{MHz}, \mathrm{CDCl}_{3}\right): \delta$ 7.20-7.18 (m, 1H, Ar-H), 6.94-6.89 (m, 2H, Ar-H), 4.73$4.56\left(\mathrm{~m}, 2 \mathrm{H}, \mathrm{CH}_{2} \mathrm{NO}_{2}\right), 4.32-4.28(\mathrm{~m}, 1 \mathrm{H}, \mathrm{Ar}-\mathrm{H}), 2.95\left(\mathrm{~d}, J=6.5 \mathrm{~Hz}, 2 \mathrm{H}, \mathrm{CH}_{2}\right), 2.14\left(\mathrm{~s}, 3 \mathrm{H}, \mathrm{CH}_{3}\right)$ ppm; ${ }^{13} \mathrm{C}$ NMR (75 MHz, $\left.\mathrm{CDCl}_{3}\right): \delta 205.0(\mathrm{C}=\mathrm{O}), 141.6$ (Cquat), $127.0(\mathrm{CH}), 125.4 \mathrm{~N}(\mathrm{CH})$, $124.6(\mathrm{CH}), 79.6\left(\mathrm{CH}_{2}\right), 46.7\left(\mathrm{CH}_{2}\right), 34.4(\mathrm{CH}), 30.2\left(\mathrm{CH}_{3}\right) \mathrm{ppm}$.

\section{Acknowledgements}

We are grateful for the financial support from National Natural Science Foundation of China (20802075) and the Chinese Academy of Sciences.

\section{References and Notes}

1. For an overview on asymmetric Michael additions, see: (a) Christoffers, J. Eur. J. Org. Chem. 1998, 7, 1259. (b) Sibi, M. P.; Manyem, S. Tetrahedron 2000, 56, 8033. (c) Krause, N.; Hoffmann-Roder, A. Synthesis 2001, 171. (d) Berner, O. M.; Tedeschi, L.; Enders, D. Eur. J. Org. Chem. 2002, 1877. (e) Christoffers, J.; Baro, A. Angew. Chem. Int. Ed. 2003, 42, 1688. (f) Notz, W.; Tanaka, F.; Barbas III, C. F. Acc. Chem. Res. 2004, 37, 580. (g) Ballini, R.; Bosica, G.; Fiorini, D.; Palmieri, A.; Petrini, M. Chem. Rev. 2005, 105, 933. (h) Xu, L.-W.; Xia, C.-G. Eur. J. Org. Chem. 2005, 633. (i) Almasi, D.; Alonso, D.A.; Najera, C. Tetrahedron: Asymmetry 2007, 18, 299. (j) Vicario,J. L.; Badia, D.; Carrillo, L. Synthesis 2007, 2065. (k) Sulzer-Mossé, S.; Alexakis, A. Chem. Commun. 2007, 3123. (1) Tsogoeva, S. B. Eur. J. Org. Chem. 2007, 1701. (m) Nising, C. F.; Bräse, S. Chem. Soc. Rev. 2008, 37, 1218.

2. Selected examples: (a) Sammis, G. M.; Jacobsen, E. N. J. Am. Chem. Soc. 2003, 125, 4442. (b) Mita, T.; Sasaki, K.; Kanai, M.; Shibasaki, M. J. Am. Chem. Soc. 2005, 127514.

3. For selected examples on organocatalytic asymmetric Michael addition of aldehydes to nitroolefins, see: (a) Betancort, J. M.; Barbas III, C. F. Org. Lett. 2001, 3, 3737. (b) Wang,W.; Wang, J.; Li, H. Angew. Chem. Int. Ed. 2005, 44, 1369. (c) Hayashi, Y.; Gotoh, H.; Hayashi, T.; Shoji, M. Angew. Chem. Int. Ed. 2005, 44, 4212. (d) Lalonde, M.P.; Chen, Y.; Jacobsen, E. N. Angew. Chem. Int. Ed. 2006, 45, 6366. (e) Palomo, C.; Vera, S.; Mielgo, A.; GómezBengoa, E. Angew. Chem. Int. Ed. 2006, 45, 5984. (f) Barros, M. T.; Phillips, A. M. F. Eur. J. Org. Chem. 2007, 178. (g) Zhu, S.; Yu, S.; Ma, D. Angew. Chem. Int. Ed. 2008, 47, 545. (h) Wiesner, M.; Revell, J. D.; Wennemers, H. Angew. Chem. Int. Ed. 2008, 47, 1871. (i) Chi, Y.; 
Guo, L.; Kopf, N. A.; Gellman, S. H. J. Am. Chem. Soc. 2008, 130, 5608. (j) Enders, D.; Wang, C.; Bats, J. W. Angew. Chem. Int. Ed. 2008, 47, 7539.

4. For selected examples on organocatalytic asymmetric Michael addition of ketones to nitroolefins, see: (a) List, B.; Pojarliev, P.; Martin, H. J. Org. Lett. 2001, 3, 2423. (b) Enders, D.; Seki, A. Synlett 2002, 26. (c) Ishii, T.; Fujioka, S.; Sekiguchi, Y.; Kotsuki, H. J. Am. Chem. Soc. 2004, 126, 9558. (d) Mase, N.; Watanabe, K.; Yoda, H.; Takabe, K.; Tanaka, F.; Barbas III, C. F. J. Am. Chem. Soc. 2006, 128, 4966. (e) Luo, S.; Mi, X.; Zhang, L.; Liu, S.; Xu, H.; Cheng, J. Angew. Chem. Int. Ed. 2006, 45, 3093. (f) Pansare, S. V.; Pandya, K. J. Am. Chem. Soc. 2006, 128, 96245. (g) Ni, B.; Zhang, Q.; Dhungana, K.; Headley, A. D. Org. Lett. 2009, 11, 1037. (h) Xue, F.; Zhang, S.-L.; Duan, W.-H.; Wang. W. Adv. Synth. Catal. 2008, 350, 2194. (i) Rasappan, R.; Reiser. O. Eur. J. Org. Chem. 2009, 1305.

5. For selected examples on organocatalytic asymmetric conjugate addition of 1, 3-dicarbonyl compounds to nitroolefins, see: (a) Okino, T.; Hoashi, Y.; Takemoto, Y. J. Am. Chem. Soc. 2003, 125, 12672. (b) Li, H.; Wang, Y.; Tang, L.; Deng, L. J. Am. Chem. Soc. 2004, 126, 9906. (c) Wang, J.; Li, H.; Duan, W.; Zu, L.; Wang, W. Org. Lett. 2005, 7, 4713. (d) McCooey, S. H.; Connon, S. J. Angew. Chem. Int. Ed. 2005, 44, 6367. (e) Li, H.M.; Wang, Y.; Tang, L.; Wu, F.H.; Liu, X.F.; Guo, C.Y.; Foxman, B. M.; Deng, L. Angew. Chem. Int. Ed. 2005, 44, 105. (f) Terada, M.; Ube, H.; Yaguchi, Y. J. Am. Chem. Soc. 2006, 128, 1454. (g) Lubkoll, J.; Wennemers, H. Angew. Chem. Int. Ed. 2007, 46, 6841. (h) Malerich, J. P.; Hagihara, K.; Rawal, V. H. J. Am. Chem. Soc. 2008, 130, 14416. (i) Wang, C.; Zhang, Z.; Dong, X.; Wu, X. Chem. Commun. 2008, 1431. (j) Li, X.J.; Liu, K.; Ma, H.; Nie, J.; Ma, J.A. Synlett 2008, 3242 (k) Gao, P.; Wang, C.G.; Wu, Y.; Zhou, Z.H.; Tang, C.C. Eur. J. Org. Chem. 2008, 4563. (1) Luo, J.; Xu, L.W.; Hay, R. A. S.; Lu, Y. X. Org. Lett. 2009, 11, 437.

6. Huang, H. B.; Jacobsen, E. N.; J. Am. Soc. Chem. 2006, 128, 7170.

7. Tanmay, M.; Zhao, C. G. Angew. Chem. Int. Ed. 2008, 47, 7714.

8. Tsogoeva, S. B.; Yalaov D. A.; Hateley, M. J.; Weckbecker, C.; Huthmacher, K. Eur. J. Org. Chem. 2005, 4995

9. For selected Michael reactions involving acetone as donor, see: (a) Cao, C.-L.; Ye, M.-C.; Sun, X.-L.; Tang, Y. Org. Lett. 2006, 8, 2901. (b) Cao, Y.-J.; Lai, Y.-Y.; Wang, X.; Li, Y.-J.; Xiao, W.-J. Tetrahedron Lett. 2007, 48, 21. (c) Vishnumaya; Singh, V. K. Org. Lett. 2007, 9 , 1117. (d) Jiang, X.X.; Zhang, Y.F; Chan, Albert S. C.; Wang, R. Org. Lett. 2009, 11, 153. (e) Kokotosa, C. G.; Kokotosa, G. Adv. Synth. Catal. 2009, 351, 1355. (f) Gu, Q.; Guo, X.T.; Wu, X.Y. Tetrahedron 2009, 65, 5265. (g) Liu, S.-P.; Zhang, X.-J.; Lao, J.-H.; Yan, M. ARKIVOC. 2009, (vii), 268.

10. (a) Tillman, A. L.; Ye, J. X.; Dixon, D. J. Chem. Commun. 2006, 11913. (b) McCooey, S. H.; Connon, S. J. Angew. Chem., Int. Ed. 2005, 44, 6367.

11. (a) Taylor, M. S.; Jacobsen, E. N. Angew. Chem. Int. Ed. 2006, 45, 1520. (b) Doyle, A. G.; Jacobsen, E. N. Chem. Rev. 2007, 107, 5713

12. For reviews on asymmetric enamine and iminium ion catalysis, see: (a) List, B. Acc. Chem. Res. 2004, 37, 548. (b) Mukherjee, S.; Yang, J. W.; Hoffmann, S.; List, B. Chem. Rev. 2007, 
107, 5471. (c) Lelais, G.; MacMillan, D. W. C. Aldrichimica Acta. 2006, 39, 79. (d) Erkkil, V. A.; Majander, I.; Pihko, P. M. Chem. Rev. 2007, 107, 5416.

13. Mei, K.; Zhang, S. L.; He, S. T.; Li, P.; Jin, M.; Xue, F.; Luo, G. S.; Zhang, H. Y.; Song, L. R.; Duan, W. H.; Wang, W. Tetrahedron Lett 2008, 49, 2681.

14. (a) Chen, Y. Z.; Lin, H.; Xu, X. Y.; Xia, S. W.; Wang, L. X. Adv. Synth. Catal. 2008, 350, 426. (b) Chen, Y. Z.; Xu, J. G.; Xu, X. Y.; Xia,Y.; Lin, H.; Xia, S. W.; Wang, L. X. Tetrahedron: Asymmetry 2007, 18, 2537. (c) Wang, L. X.; Shen, J. F.; Tang, Y.; Chen, Y.; Wang, W.; Cai, Z. G.; Du, Z. J. Org. Process Res. Dev. 2007, 11, 487. (d) Lin, H.; Chen, Y. Z.; Xu, X. Y.; Xia, S. W.; Wang, L. X.H. J. Mol. Catal. B-Enzym. 2009, 57, 1.

15. (a) Wang, L. X.; Tang, Y.; Chen, Y.; Tian, F. Eur. Pat. Appl. 1942110. (b) Wang, L. X.; Tang, Y.; Chen, Y.; Tian, F. U.S. Patent 20080249311. (c) Wang, L. X.; Tang, Y.; Chen, Y.; Tian, F. World Patent 2007028337. (d) Wang, L. X.; Tang, Y.; Chen, Y.; Tian, F.C.N..Patent ZL200510060721.2, 2007, Chem. Abstr. 2007, 146, 337870. (e) Wang, L. X.; Tang, Y.; Chen, Y.; Tian, F. C.N. Patent ZL200510061230.X, 2007; Chem. Abstr. 2007, 146, 337870. (f) Wang, L. X.; Tang, Y.; Chen, Y.; Tian, F. C.N. Patent ZL200510061231.4, 2007; Chem. Abstr. 2007, 146, 337870. (g) Wang, L. X.; Peng, Q. H.; Du, Z. J.; Lan, H. F. C.N. Patent 200610027596.X, 2006; Chem. Abstr. 2006, 148, 121716.

16. Yang, Z. G.; Liu, J.; Liu, X. H.; Wang, Z.; Feng, X. M.; Su, Z. S., Hu, C. W. Adv. Synth. Catal. 2008, 350, 2001. 